phylaxis had not been given to contacts of these patients, but only one of the "secondary" cases occurred within 30 days of the index case; the others were two months and three months apart. Although rifampicin prophylaxis is of limited value, the relatively poor compliance with the current recommendations suggests that its value is commonly reduced even further by incorrect use.

There has been concern that widespread use of rifampicin might lead to the development of rifampicin resistant strains of $H$ influenzae type $b .^{10}$ However, in our laboratory all of 120 strains of $H$ influenzae type b tested by agar dilution were inhibited by $<0: 03 \mathrm{mg} / \mathrm{l}$ rifampicin (unpublished data). Other objections to the use of rifampicin are that it sometimes fails to prevent secondary $H$ influenzae type $\mathrm{b}$ disease ${ }^{9}$ and that it is associated with significant cost and risk of toxicity. A four day course for a family of two adults and three children is \$A50 and the risk of toxicity is small if suitable precautions are observed. At present there is no alternative to rifampicin for contacts in whom its use is contraindicated - for example, pregnant women. Ceftriaxone is effective in eliminating Neisseria meningitidis from the throats of carriers. ${ }^{22}$ With the more widespread use of this agent for treating invasive $H$ influenzae type $\mathrm{b}$ disease it would be useful to determine whether it can also eliminate this organism from the throats of patients; if so, it may be an alternative to rifampicin for selected contacts.

This study has emphasised the limitations of antibiotic prophylaxis in preventing $H$ influenzae type $\mathrm{b}$ disease. A significant impact on its incidence can be achieved only by immunisation,,$^{23}$ which should be introduced as soon as possible for protection of children at risk.

1 Filice GA, Andrews JS, Hudgins MP, Fraser DW. Spread of Haemophilus influenzae. Secondary illness in household contacts of patients with $\mathrm{H}$ influenzae meningitis. Am $\mathcal{F}$ Dis Child 1978;132:578-9.

2 Ward JI, Fraser DW, Baraff LJ, Plikaytis BD. Haemophilus influenzae meningitis. A national study of secondary spread in household contacts. N Engl F Med 1979;301:122-6.
3 Glode MP, Daum RS, Goldmann DA, Leclair J, Smith A. Haemophilus influenzae type $b$ meningitis: a contagious disease of children. $B M J$ 1990;280:899-901.

4 Campbell LR, Zedd AJ, Michaels RH. Household spread of infection due to Haemophilus influenzae type b. Pediatrics 1980;66:115-7.

5 Glode MP, Daum RS, Boies EG, Ballard TL, Murray M, Granoff DM. Effect of rifampicin chemoprophylaxis on carriage eradication and new acquisition of Haemophilus influenzae type b contacts. Pediatrics 1985;76:537-42.

6 Band JD, Fraser DW, Ajello G. Prevention of Haemophilus influenzac type b disease. FAMA 1984;251:2381-6.

7 Centers for Disease Control. Prevention of secondary cases of Haemophilus influenzac type b disease. MMWR 1982;31:672-4, 679-80.

8 American Academy of Pediatrics, Committee on Infectious Diseases. Revision of recommendation for use of rifampicin prophylaxis of contacts of patients of recommendation for use of rifampicin prophylaxis of contacts of
with Haemophilus influenzae infection. Pediatrics 1984;74:301-2.

9 Bodies EG, Granoff DM, Squires JE, Barenkamp SJ. Development of Haemophilus influenzae type $b$ meningitis in a household contact treated with rifampicin. Pediatrics 1982;70:141-2.

10 Murphy TV, McCracken GH, Zweighaft TC, Hansen EJ. Emergence of rifampicin-resistant Haemophilus influenzae after prophylaxis. I Pediat 1981;99:406-9.

11 Michaels RH, Norden CW. Pharyngeal colonization with Haemophilus influenzae type b: a longitudinal study of families with a child with meningitis or epiglottitis due to $\mathrm{H}$ influenzae type $\mathrm{b}$. 7 Infect $D_{\text {ts }}$ 1977;136:2228.

12 Daum RS, Glode MP, Goldmann DA, et al. Rifampicin chemoprophylaxis fo household contacts of patients with invasive infections due to Haemophilus influenzae type b. F Pediatr 1981;98:485-91.

13 Gilsdorf JR. Dynamics of nasopharyngeal colonization with Haemophilus influenzae type b during antibiotic therapy. Pediatrics 1986;77:242-5.

14 Apert GA, Campos JM, Smith DR, Barenkamp SJ, Fleisher GR. Incidence and persistence of Haemophilus influenzae type b upper airway colonization and persistence of Haemophilus influenzae type b upper
in patients with meningitis. F Pediatr 1985;107:555-7.

15 Gilbert GL, Clements DA, Broughton AJ. Haemophilus influenzae type b infections in Victoria, Australia, 1985-1987. Pediatr Infect Dis $\mathcal{F}$ 1990;9:252-7.

16 Michaels RH, Stonebraker FE, Robbins JB. Use of antiserum agar for detection of Haemophilus influenzae type $\mathrm{b}$ in the pharynx. Pediatr Res 1975;9:513-6.

17 Gilbert GL. Epidemiology and prevention of invasive Haemophilus influenza type b infection. Aust Paediatr f 1987;23:323-7.

18 Gilbert GL. Antibiotic prophylaxis for contacts of patients with invasive infection due to Haemophilus influenzae type b. Australian College of Paediatrics Newsletter, May, 1987.

19 Michaels RH, Poziviak CS, Stonebraker FE, Norden CW. Factors affecting pharyngeal Haemophilus influenzae type b colonization rates in children. Y Clin Microbiol 1976;4:413-7.

$20 \mathrm{Li} \mathrm{KI}$, Wald ER. Use of rifampicin in Haemophilus influenzae type $r$ infections. Am F Dis Child 1986;140:381-5.

21 Shapiro ED, Wald ER. Efficacy of rifampicin in eliminating pharyngeal carriage of Haemophilus influenzae type b. Pediatr 1980;66:5-8.

22 Schwartz B, Al-Tobaiqi A, Al-Ruwais. et al. Comparative efficacy of ceftriaxone and rifampicin in eradicating pharyngeal carriage of group A Neisseria meningitidis. Lancet 1988;i:1239-42.

23 Clements DA, Gilbert GL. Immunization for prevention of Haemophilus influenzae type b infection: a review. Aust NZ F Med 1990;20:828-34.

(Accepted 12 April 1991)

\section{Audit of an official recommendation on screening for congenital dislocation of the hip}

\author{
Dai Anthony Jones, Dawn Beynon, \\ B N C Littlepage
}

Guidance on screening for congenital dislocation of the hip has been given by the Standing Medical Advisory Committee for the Central Health Services Council, which reported in 1966 and 1969.' In 1986 this advice was updated. ${ }^{2}$ The booklet was made widely available and also stimulated editorials and special articles in leading medical journals. ${ }^{3+}$ This report was published against a background of doubt that screening was effective and also evidence that the incidence of late cases was increasing despite screening.

The 1986 recommendations recognised that several health professionals may take part in the screening process, and therefore recommended that " $A$ designated officer within each Health District should keep the whole Screening programme under review and record and evaluate the incidence of cases detected late - such information should be made available to the health professionals concerned." Although the term "audit" was not widely used at the time, it is clear that this is what was being proposed-that is, feedback of the failures of the process of screening and modification of the process accordingly. We assessed the effectiveness of the distribution of the recommendation and its level of implementation.

\section{Methods and results}

A questionnaire was sent to each health district in the United Kingdom. Out of a wide range of questions asked, two were: "Have you identified a designated officer within your district to keep the whole screening programme under review?" and "Does any paediatrician or orthopaedic surgeon in your district have a special interest in congenital dislocation of the hip? (Please name.)"

Of the 216 questionnaires distributed, 152 health districts replied (70\%). Only $67(45 \%)$ of these health districts had appointed a designated officer to oversee the screening process. Of the 150 replies, 95 named clinicians who had a special interest in congenital dislocation of the hip: 75 were orthopaedic surgeons, 13 were paediatricians, one was a community physician, and the others quoted a combination of specialties.

\section{Comment}

The revised memorandum on screening for congenital dislocation of the hip was widely circulated to professional groups and health authorities accompanied by a letter from the chief medical officer or chief nursing officer $(\mathrm{C} 190(86) 17, \mathrm{CNO}(86) 13)$. A press 


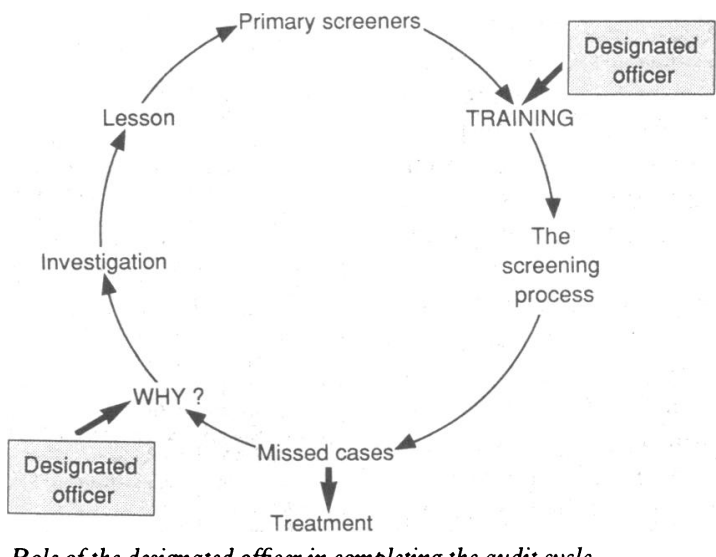

Role of the designated officer in completing the audit cycle

release from the Department of Health and Social Security and the Welsh Office drew attention to the wide distribution and to the advice provided.

The fact that in $57 \%$ of districts no designated officer had been identified is difficult to understand. Seventy per cent of the replies were able to name clinicians with a special interest in congenital dislocation of the hip and therefore it might be considered reasonable for these clinicians to have been nominated as the designated officers. The fact that most of these were orthopaedic surgeons and not paediatricians is also rather surprising because congenital dislocation of the hip has been perceived over the past 30 years to be a paediatric disease, largely because of the influence of Palmen in Sweden and Dunn in Bristol. Orthopaedic surgeons may, however, be regarded as appropriate for this role because they are called on to treat the patients for whom the screening process has failed. The designated officer, as well as taking part in audit, must also be involved in training staff in both primary and secondary screening (figure).

This study identified the wide variation in response that can occur to an official piece of advice. That no designated officer had been named in over half the health districts that replied is a deficit in the process of checking that advice given is acted on. This might also be true for official advice given in other fields of medicine. We consider it essential that this matter is urgently corrected and would suggest that the designated officer should be responsible for audit and training as indicated in the figure.

We thank the chief administrative medical officers and their staff who replied to our questionnaire.

1 Standing Medical Advisory Committee for the Central Health Services Council, Secretary of State for Social Services. Screening for the detection of congenital dislocation of the hip in infants. London: Department of Health and Social Security, 1969.

2 Standing Medical Advisory Committee, Standing Nursing and Midwifery Advisory Committee for the Secretaries of State for Social Services and fo Wales. Screening for the detection of congenital dislocation of the hip. London Department of Health and Social Security, 1986.

3 Macfarlane A. Screening for the detection of congenital dislocation of the hip BMf 1987;294:1047.

4 Anonymous. Screening for the detection of congenital dislocation of the hip. Arch Dis Child 1986;61:921-6.

5 Catford JC, Bennett GC, Wilkinson JA. Congenital hip dislocation: an increasing and still uncontrolled disability. BMF 1982;285:1527-30.

(Accepted 22 March 1991)

\section{ONE HUNDRED YEARS AGO}

It is extremely unfortunate that those who control the supply of necessaries to large public and private establishments do not adopt systematic and reliable means of assuring themselves that the nature and quality of their contractors'goods are what they are represented to be. The almost universal want of attention to these matters is not generally known among the public; where it is known it can hardly be adequately appreciated. The immense quantities of food of all kinds which are supplied daily to our hospitals, workhouses, prisons, and other large public institutions are, as a general rule, taken in on faith. No check of any kind is placed upon the contractors, and the result of this want of care is what might naturally be expected. As we have pointed out when dealing with this subject on previous occasions, the proper examination of contractors' goods is exceedingly rare, and when any such examinations have been made it is still more rare for the facts to be published. Wholesale "manipulation," carried on practically without the fear of detection, is a proceeding which pays extremely well, and those who indulge in it no doubt "know their business." The supply of milk and of butter may be cited as examples. These supplies may be most ingeniously manipulated, and it is within our knowledge that they are so. By judicious admixture of "separated" milk with "whole" milk a product is obtained which contains the lowest possible amount of fat - that is, such an amount as is found only in the milk of single cows yielding the very poorest quality. Mixtures of butter and margarine are prepared containing comparatively small percentages of the latter, but still quite large enough to yield handsome profits to the dealers, and defying detection by all chemical tests excepting that of thorough and complete analysis.
Cocoa is another article of which very large amounts are supplied to public institutions, and it is highly probable that the major portion of these supplies consists of so-called "cocoa powder," grossly adulterated with starch and sugar. Instances might be multiplied; and it is to be noted that public institutions are by no means the only "happy hunting grounds" of the adulterating contractor and dealer. Large private establishments, such as clubs, hotels, and restaurants, are liable to the same frauds; but they are probably not so badly off as public establishments, where it is seldom anybody's business to examine the supplies, and where, accordingly, no official or manager is likely to suffer personally through the passing of inferior goods.

The necessity of systematically examining the food supplies is not, however, the only point to which we desire that attention should be directed. Stores of all kinds delivered under contract are subjected to fraudulent practices of a similar character to those which are applied to food. We believe that all kinds of articles supplied under contract to some of the Government departments are now constantly examined by competent experts, with the result that a considerable number are condemned. It would surely be highly desirable that this system of control should be widely extended.

We have reason to believe that the drugs supplied to public establishments-especially to prisons and workhouses - are often of very inferior quality. This is a matter which is not less serious than the adulteration of the food supplies. The great difficulty is to bring home the importance of the whole matter to the various governors and bodies who are, or ought to be, responsible. (British Medical fournal 1891;i:812) 